\title{
High Way Vehicle Speed Control \& Automatic Breaking System
}

\author{
Avvaru Subramanyam ${ }^{1}$, K.Satya Rajesh ${ }^{2}$, L.Bharhav Kumar, ${ }^{3}$ \\ ${ }^{1,3}$ Assistant Professor, Dept. of ECE,${ }^{2}$ IV Year B.Tech., Dept. of E.C.E. \\ Mekapati Rajamohan Reddy Institute of Technology and Science, Udayagiri, Andhra Pradesh, India.
}

\begin{abstract}
Every year, we find more and more road accidents due to increased traffic on the roads, and if you see the statistics, you will find that the causalities are more every year than that of 1970 Indo-Pak war. Experts say, increased motorist population, long working hours and stressful life are the major reasons for the rise in road accidents. The factors are beyond one's control, but if we could alert the driver on the highway, could save many precious lives. We haven't presented a very complex solution for this; neither claimed to be expert traffic controllers. Usually fixing some sort of transmitter system on the highways which can detect the speed of the vehicle and convey to the driver that he is not in the permitted speed limit in a particular area is quite common and expensive. As a simple solution, we have pre installed low cost transmitters on required locations to convey the speed limit information, even further, if the automatic breaking system could be actuated on over speed is a decent approach.

We have designed and developed a prototype system of a transmitter and receiver modules. Receiver(s) is (are) kept in the vehicle(s) and transmitter(s) are set up on the road side. As it is a prototype, we have not used any speed sensing devices, instead four switches are used as alternative. The four switches represents or assumes various speed limits say limit 80 switch, limit40 switch, limit20 switch and no horn area. Whenever these switches are pressed, they will transmit the corresponding alert message to the driver so that the driver can handle the situation sensibly. Receiver section has a $2 \times 16$ LCD, which will display the alert message, and there exists microcontroller to receive the data and activate the relay circuitry to give a buzzer and to control the speed of the dc motor which acts as a prototype for automatic braking and vehicle speed reduction mechanism.

Key words: Road accidents, alerting the driver by message, transmitter and receiver system, microcontroller, speed limits, no horn area, buzzer.
\end{abstract}

\section{Introduction}

As per the surveys held, statistics stated by various governments of different countries and states, every year, road accidents rate is continuously climbing up due to increased traffic on the roads. The causality rate is progressively increasing year by year. Experts say that the increase in motorist population is one of the main reason for it. Long working hours is one more major concern of the city people. The life is more and more stressful now a days. The above three points mentioned are predominantly known as the primary and most common reasons for the road accidents, and especially on highways in most of the states.

If we could alert the vehicle driver on the highway much ahead in time, could save many precious lives and leads to reduction in death rate due to highway accidents. It is quite common to fix up some sort of system on the highways which can detect the speed of the vehicle and convey to the driver that he is not in the permitted speed limit in a particular area. But in our solution, depending on the geographical location, transmitter systems are permanently set up to transmit the speed limit and no horn area kind of information, even further, if the automatic breaking system could be actuated on over speed would be an excellent feature. We have tried to attempt a simple solution which is of much similar to a prototype of its kind. We have designed and developed a prototype system of a transmitter and receiver modules.

\section{Design Methodology}

The main objective of this project is to alert the driver at danger zones and to activate the breaking system, if it is needed. This can be done easily by using RF communication system. The RF transmitter sections are placed at each and every danger zone such as school zones, speed limit areas and hospital areas etc. The RF receiver sections are placed each and every vehicles. The RF Transmitter and receiver will communicate by using electromagnetic waves in free space medium.

The Transmitter section contains the information of limitations of particular area and it transmits the information signals in to the air. The transmitter section consists of switches, encoder, RF transmitter . The Rx receiver section consists of $\mathrm{Rf}$ receiver, micro controller, dc- motor, LCD display, buzzer.

These sections are placed in vehicles, when the vehicle enters in to the transmitter region it receives the data signals from the transmitter. The data signal is in the form of electromagnetic waves. The RF receiver converts the them in to electrical data signals. The micro controllers is coded in such a way that it activates the 
particular action corresponding to the received signal. Coming to the power supply, this project uses regulated $5 \mathrm{~V}, 750 \mathrm{~mA}$ power supply. 7805 three terminal voltage regulator is used for voltage regulation. Bridge type full wave rectifier is used to rectify the ac output of secondary of $230 / 18 \mathrm{~V}$ step down transformer.

\section{a. Transmitter section}

\section{Block Diagrams}

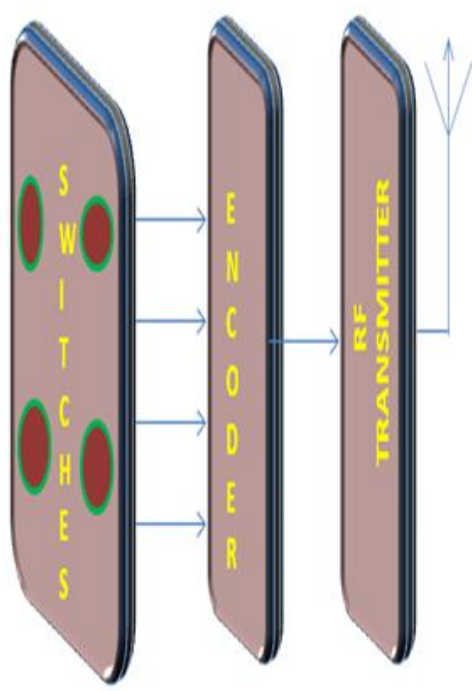

Fig.l.Block diagram of transmitter section

The block diagram of the transmitter section consists of switches connected to an encoder and RF transmitter. Four switches are provided for giving details regarding the speed limit of a particular zone in the highway. Switch 1 represents speed limit of 80kmph, switch 2 represents a speed limit of $40 \mathrm{kmph}$, switch 3 reprents a maximum speed limit of $20 \mathrm{kmph}$ and finally switch 4 corresponds to a zone where blowing horn is prohibited. For the purpose of prototype we have combined 4 transmitters with above individual feature into a single transmitter to lower the prototype development cost.

\section{b. Receiver section}

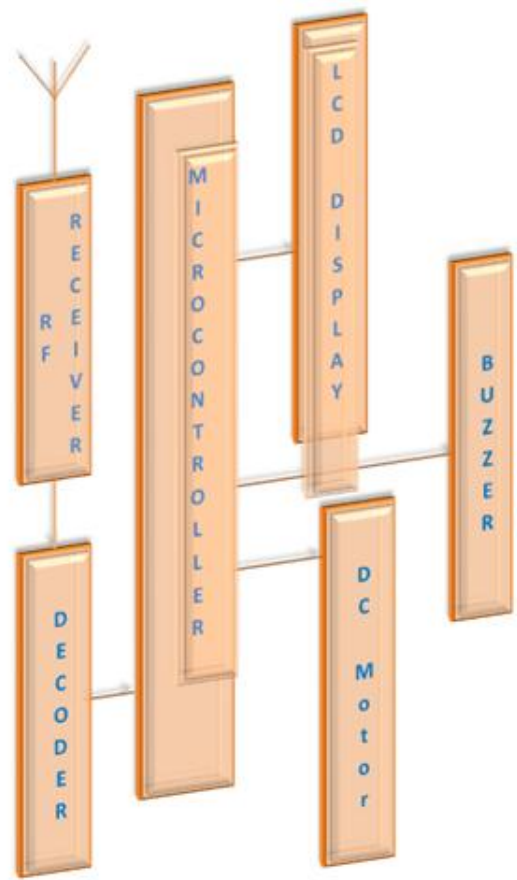

Fig.2.Block diagram of receiver section

The block diagram of the receiver section consists of a receiving antenna, a microcontroller, an LCD display, a DC motor and a buzzer as shown in the diagram. The antenna receives the transmitted speed limit information or no horn zone related data. It is fed to the decoder and the decoder decodes the information and 
gives it to the microcontroller. Depending on the received information, i.e. whether speed limit or no horn zone data, the microcontroller controls the speed of the DC motor and or activates the buzzer. The same information can also be seen on the 16x2 LCD display also. By observing the above or hearing the beep sound of the buzzer, the driver gets alerted.

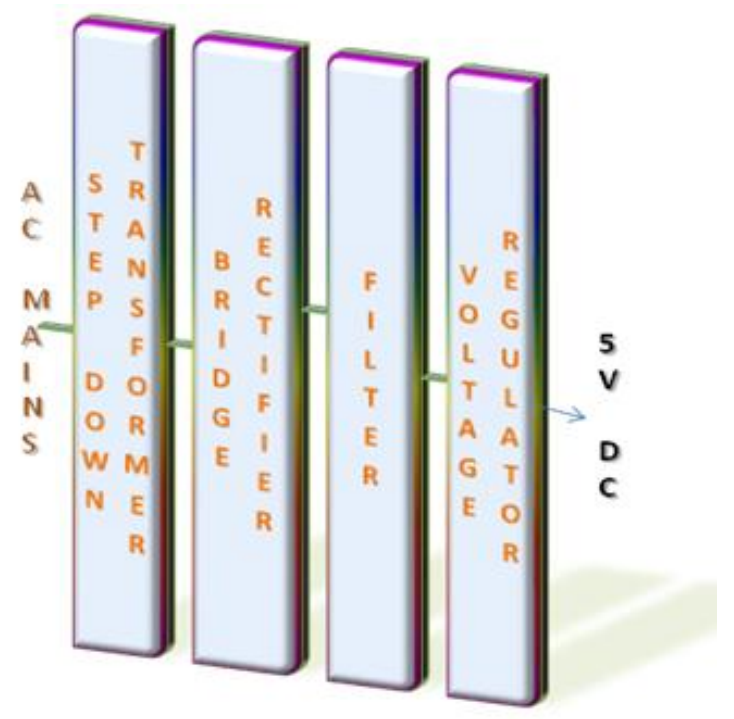

Fig 3. Block diagram of the power supply section

The block diagram of the power supply section consists of a step down transformer, a bridge rectifier, a filter and a voltage regulator.The a.c. input i.e., $230 \mathrm{~V}$ from the mains supply is given to the step down transformer. The step down transformer steps down the applied $230 \mathrm{v}$ ac to $12 \mathrm{~V}$ ac. The $12 \mathrm{v}$ ac is applied to the bridge rectifier. The output obtained from the rectifier is a pulsating d.c voltage. So in order to get a pure d.c voltage, the output voltage from the rectifier is fed to a filter to remove any a.c components present even after rectification. Now, this voltage is given to a voltage regulator to obtain a pure constant dc voltage. The voltage regulator produces a constant $5 \mathrm{v}$ dc output.

\section{Software Tools}

For working with microcontroller code development aspect, we have used Keil compiler. To dump the footprint of the application, Proload is used.

\section{a. Keil Compiler}

Keil compiler is a software used where the machine language code is written and compiled. After compilation, the machine source code is converted into hex code which is to be dumped into the microcontroller for further processing. Keil compiler also supports $\mathrm{C}$ language code.

\section{b. Proload}

Proload is a software which accepts only hex files. Once the machine code is converted into hex code, that hex code has to be dumped into the microcontroller placed in the programmer kit and this is done by the Proload. Programmer kit contains a microcontroller on it other than the one which is to be programmed. This microcontroller has a program in it written in such a way that it accepts the hex file from the keil compiler and dumps this hex file into the microcontroller which is to be programmed. As this programmer kit requires power supply to be operated, this power supply is given from the power supply circuit designed above. It should be noted that this programmer kit contains a power supply section in the board itself but in order to switch on that power supply, a source is required. Thus this is accomplished from the power supply board with an output of 12 volts or from an adapter connected to $230 \mathrm{~V}$ AC.

\section{c. ExpressPCB}

We have used Express PCB software for laying out the circuitry on to a printed circuit board. Easy to learn and use. For the first time, designing circuit boards is simple for the beginner and efficient for the professional. Learning the ExpressPCB software is fast because of its familiar user interface. Best of all, its Free. 


\section{a. RF Module}

\section{Hardware Details}

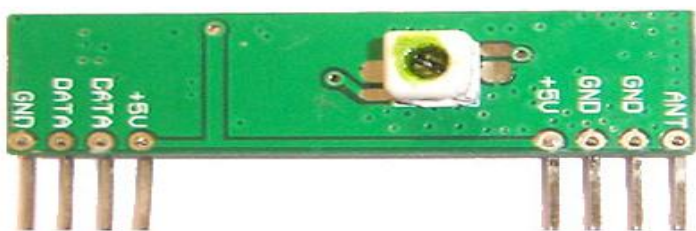

Fig 4. RF Module sample

A RF Module is a small circuit pre built and tested. They comes in Pair. One is RX or the receiver and other is a TX or Transmitter. The one we will be using is a low cost module of about Rs. 300 (US\$ 6.00) per pair in India. They have reasonable range and works very good.

As you can see they have very low pin count. This is because they are highly modular in design. The transmitter module consists of an antenna, power supply and data input pins (Vcc, DATA, GND). Whereas the Receiver module consists of an antenna, power supply pins and data output pins (VCC, GND, DATA).

The HT12E encoder is a 18 pin DIP IC. The 212 encoders are a series of CMOS LSIs for remote control system applications. They are capable of encoding information which consists of $\mathrm{N}$ address bits and 12_N data bits. Each address/data input can be set to one of the two logic states. The programmed addresses/ data are transmitted together with the header bits via an RF or an infrared transmission medium upon receipt of a trigger signal. The capability to select a TE trigger on the HT12E or a DATA trigger on the HT12E further enhances the application flexibility of the $2^{12}$ series of encoders.

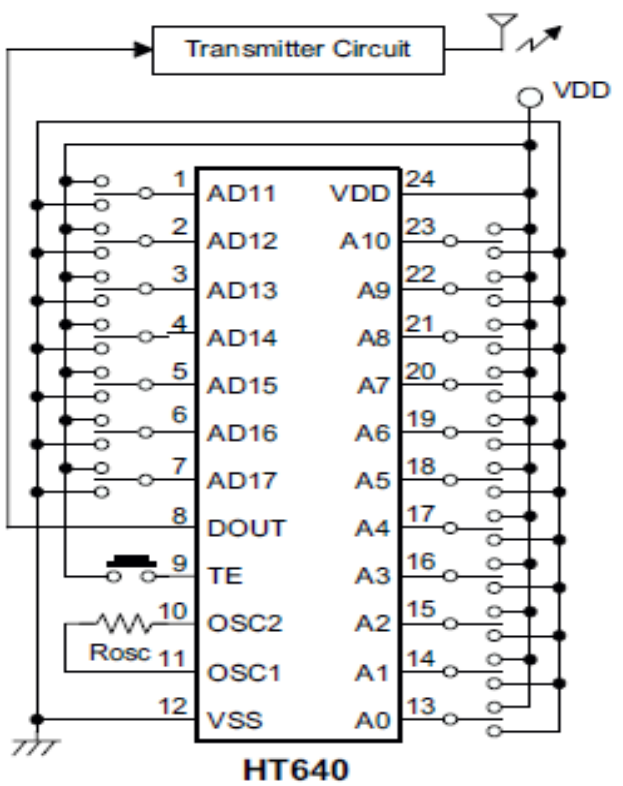

Fig. 5. Basic application circuit of Ht640 Encoder

The HT12D decoder is a 18 pin DIP IC. The 212 decoders are a series of CMOS Large Scale Integrations for remote control system applications. They are paired with Holtek's 212 series of encoders.

A pair of encoder/decoder with the same number of addresses and data format should be chosen for proper operation. The decoders receive serial addresses and data from a programmed 212 series of encoders that are transmitted by a carrier using an RF or an IR transmission medium. They compare the serial input data three times continuously with their local addresses. If no error or unmatched codes are found, the input data codes are decoded and then transferred to the output pins.

The VT pin also goes high to indicate a valid transmission. The $2^{12}$ series of decoders are capable of decoding information that consists of $\mathrm{N}$ bits of address and 12_N bits of data. Of this series, the HT12D is arranged to provide 8 address bits and 4 data bits

We have used AT89S52 micro controller. Its features are best given in the above figure. We have implemented an algorithm in its flash memory to drive the DC Motor according to information received by the Rf Receiver module. 


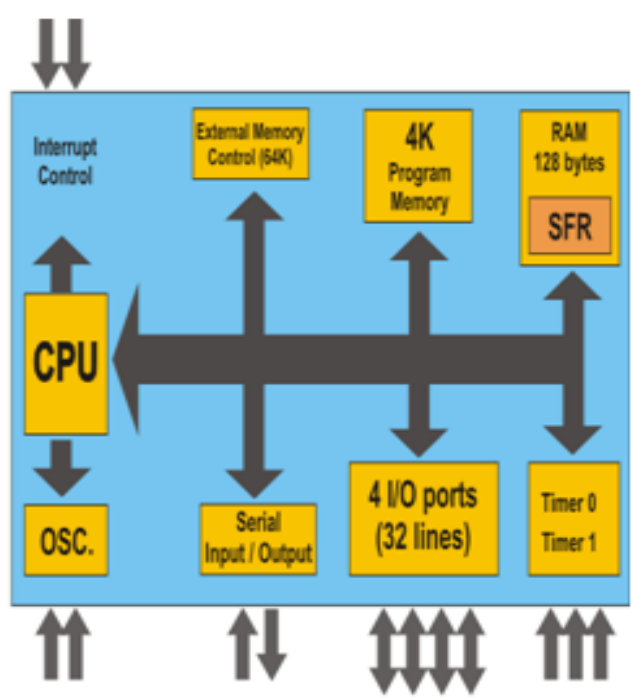

Fig.6. 8051 Family Internal blocks

\section{Experimental Results}

The results obtained after the development of the individual modules are presented in the form of photographs below.

The RF transmitter is placed in speed limited areas over the highways. This transmitters transmits the information signals continuously in to the air. The four switches represent the four different zones.

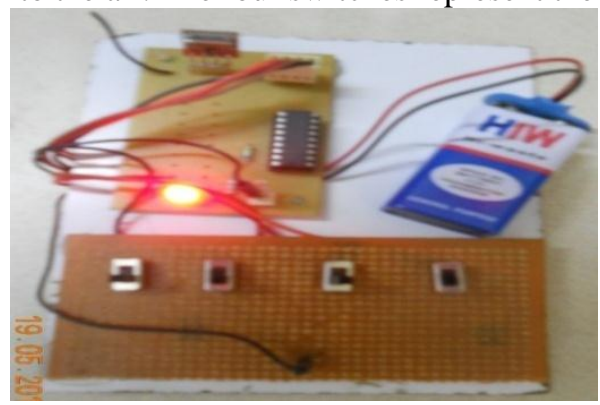

Photograph 1.RF transmitter block

8052 is an 8-bit processor, meaning that the CPU can work on only 8 bits of data at a time. This micro controller is interfaced with the breaking system, display unit, buzzer system and coded in such a way that to activate theis units based on the received information signal from the transmitter.

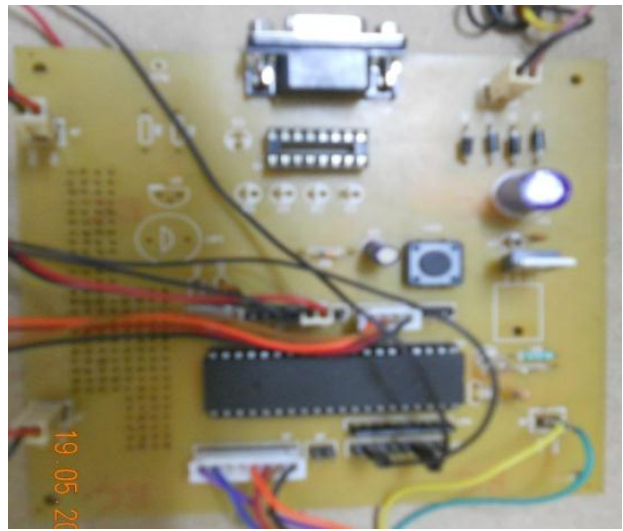

Photograph 2.8052 micro controller board

LCD stands for Liquid Crystal Display. LCD is finding wide spread use replacing the LEDs . LCD screen consists of two lines with 16 characters each. Each character consists of $5 x 7$ dot matrix. 


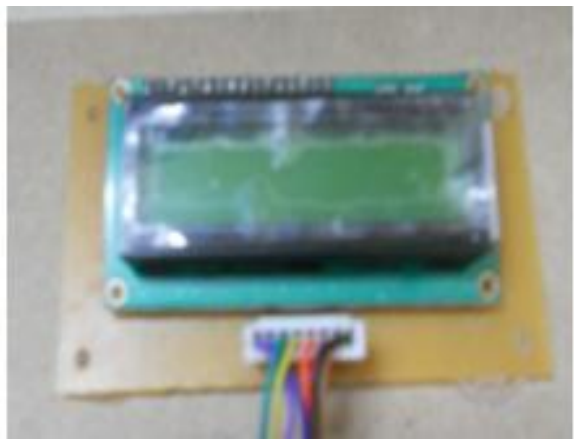

Photograph 3. LCD display

The RF receiver is placed in vehicles, when the vehicle entre in to the range of the transmitter it receives the information signals passes to the microcontroller .

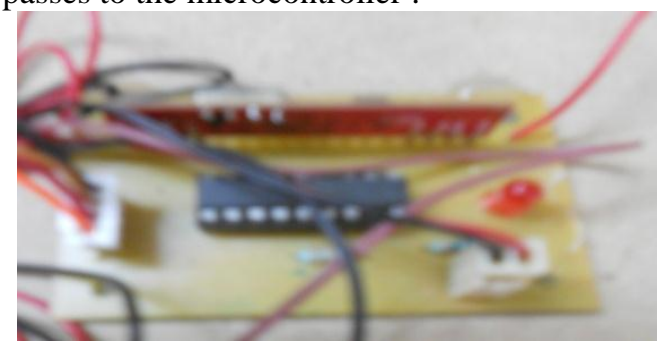

Photograph 4. receiver section

The dc motor is connected to micro controller which shows the variations in the speed according the breaking system instruction.

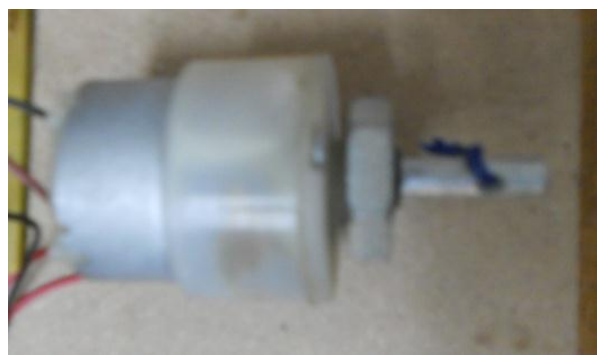

Photograph 5. :DC motor

The dc motor driver circuit is used to drive the DC motor effectively. The motor driver is connected to the p3 port of the micro controller.

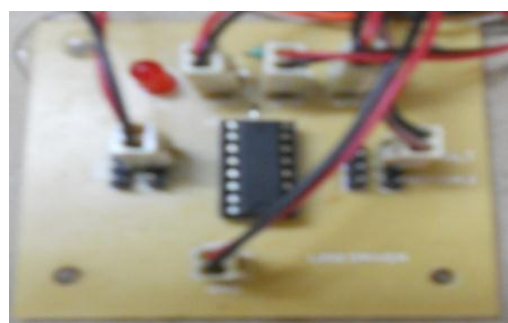

Photograph 6. DC motor driver circuit

The buzzer is used for indicating the driver when the vehicle exceeds the limitations of the zone. The buzzer section is also used to indicate the horn . 

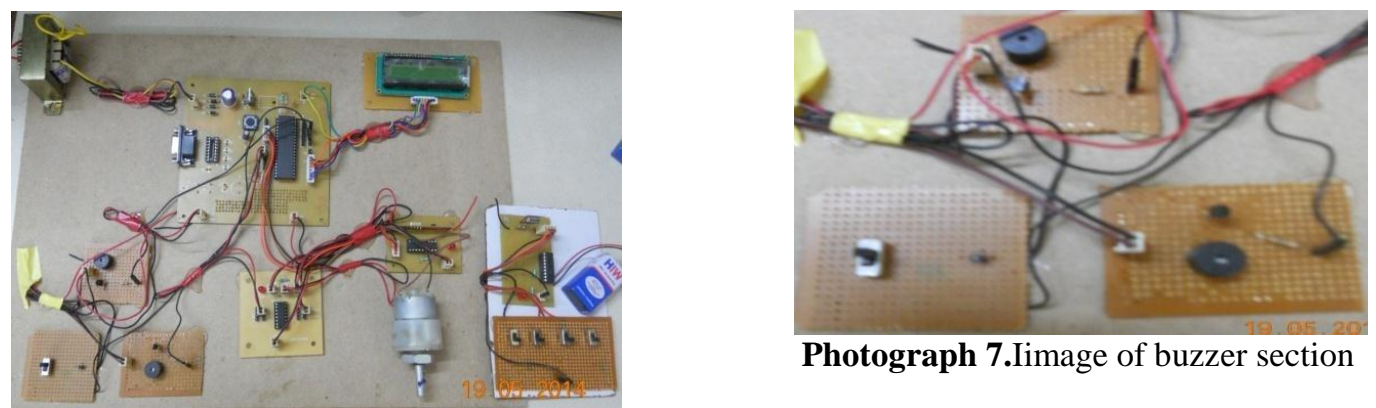

Photograph 7.Iimage of buzzer section

Photograph 8. Snap shot of entire prototype of high way speed sensing and automatic breaking system

Thus the project was designed and tested successfully by using RF Communication system. Secondly using highly advanced IC's and with the help of growing technology the project has been implemented successfully.

\section{Conclusion}

The prototype work "HIGWAY VEHICLE SPEED CONTROL AND AUTOMATIC BRAKING SYSTEM" has been successfully designed and tested. Integrating features of all the components have used developed it .Presence of every module has been reasoned out and placed carefully thus contributing the best working of the unit. Secondly using highly advanced IC's and with the help of growing technology the project has been implemented successfully.

\section{References}

[1]. Automated emergency Brake systems: Technical requirements, costs and benefits. C Grover, I Knight,I Simmons, G Couper, P Massie and B Smith, PPR 227, TRL Limited

[2]. Bishop, R. (2005) Intelligent Vehicles Technology and Trends, Artech House.

[3]. Sussman, J. M. (1993) Intelligent vehicle highway systems: Challenge for the future, IEEE Micro, 1(14-18), pp. 101-104.

[4]. Autonomos Intelligent Cruise Control. Petros A,Member, IEEE, and C.C. Chien, IEEE Transactions on Vehicular Technology, vol 42, No.4, Nov 1993.

[5]. R. E. Fenton, “A Headway safety policy for automated highway operations” IEEE Transactions on Vehicular Technology, VT-28, Feb. 1979.

\section{Biographies}

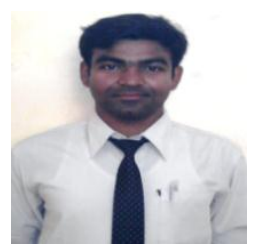

Avvaru Subramanyam, received the B.Tech. degree in Electronics and Communication Engineering from Jawaharlal Nehru Technological University, Hyderabad in 2007 \& M.Tech. degree in Embedded Systems from JNTU, Anantapur in 2011. Currently, he is an Assistant Professor of Electronics and Communication Engineering in Mekapati Rajamohan Reddy Institute of Technology and Science, Udayagiri, SPSR Nellore District, Andhrapradesh. His research areas include Digital design, Communications and embedded system design. He also qualified for the award of Junior Research Fellowship (JRF) \& Lectureship (LS) by UGC NET. The Author may be reached at avvarusubramanyam@outlook.com

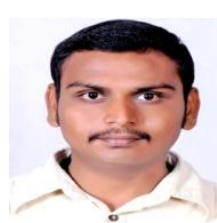

K.Satya Rajesh, a IV year B.Tech student in Department of ECE in Mekapati Rajamohan Reddy Institute of Technology and Science, Udayagiri. His intereting areas are Embedded Systems and VLSI Design. He also presented papers in various conferences and attended diversified workshops like RoboWalk etc.

His mail id is Krajeshy2k@gmail.com.

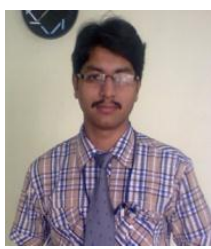

L.Bhargav Kumar, received B.E. degree from JNTU Hyderabad in 2007 and M.Tech. degree in VLSI System Design from JNT University, Anantapur in 2012. He is currently working as an Assistant Professor in MRRITS, Udayagiri. His interesting fields of research are VLSI, Image processing, digital design and Embedded system design. Mail:

1.bhargavkumar@gmail.com. 